\title{
Fusion Segmentation Method Based on Fuzzy Theory for Color Images
}

\author{
Jing Zhao ${ }^{\text {a }}$, Guoman Huang ${ }^{\text {a,*, Jiaqi Zhang }}{ }^{\text {a }}$ \\ ${ }^{a}$ Key Laboratory of Geo-Informatics of State Bureau of Surveying and Mapping, Chinese Academy of Surveying \& Mapping, \\ Beijing, China - (755800255@qq.com, huang.guoman@casm.ac.cn, sddmzhang@163.com)
}

\section{Commission III, WG III/6}

KEY WORDS: Fuzzy set theory, Fusion segmentation,Two-dimensional histogram, Uncertainty, Modeling, Membership function

\begin{abstract}
:
The image segmentation method based on two-dimensional histogram segments the image according to the thresholds of the intensity of the target pixel and the average intensity of its neighborhood. This method is essentially a hard-decision method. Due to the uncertainties when labeling the pixels around the threshold, the hard-decision method can easily get the wrong segmentation result. Therefore, a fusion segmentation method based on fuzzy theory is proposed in this paper. We use membership function to model the uncertainties on each color channel of the color image. Then, we segment the color image according to the fuzzy reasoning. The experiment results show that our proposed method can get better segmentation results both on the natural scene images and optical remote sensing images compared with the traditional thresholding method. The fusion method in this paper can provide new ideas for the information extraction of optical remote sensing images and polarization SAR images.
\end{abstract}

\section{INTRODUCTION}

Image segmentation refers to the process of dividing the image into different regions with different features. It aims to extract the object of interest from an image. Image segmentation is the basis of image interpretation and image analysis. Color image processing has been widely used in image retrieval, remote sensing image analysis, medical image analysis [1-3] since color images can provide more information than gray images.

Most segmentation techniques (such as thresholding methods [4], edge-based methods [5], region-based methods [6], and hybrid methods [7], etc.) for gray images can also be extended to segmentation of color images. We can apply the gray image segmentation method on each color channel of the color image and then combine the results from the three channels in a certain way, the final segmentation result is obtained.

The most classic segmentation method for gray images is the method based on the threshold of the histogram. Otsu method [8] determines the threshold by calculating the maximum interclass variance, which is widely used because of its simple calculation and high timeliness. However, the traditional Otsu method ignores the pixels' neighborhood information and has bad anti-noise performance. Therefore, Liu et al proposed the two-dimensional Otsu algorithm [9], in which the twodimensional histogram is built by the intensity of the pixel and the average intensity of its neighborhood pixels and the thresholds for segmentation (the threshold of the target pixel' $s$ intensity and the threshold of the average intensity of its neighborhood) is determined by calculating the maximum interclass variance. The thresholding method is inherently a hard decision method. Since there are uncertainties when labeling the pixels around the threshold, the hard decision method tends to produce erroneous segmentation results.

In 1965, Dr. L.A.Zadeh proposed the fuzzy set theory [10]. It is an effective tool for modeling and reasoning on uncertainties. This paper proposes a fusion segmentation method for color image based on fuzzy set theory. The mathematical model is established for the uncertainty of the pixel class around the threshold under a single color channel. Then the segmentation result is obtained by the maximum membership principle. The experimental results show that the proposed method can achieve better segmentation results compared with the traditional twodimensional Otsu method.

\section{BASIS OF COLOR IMAGE AND SEGMENTATION}

\subsection{Basis of color image}

In digital image processing, the most commonly used color model is the RGB (red, green, blue) model. The RGB model is based on the Cartesian coordinate system, and all colors are represented by the three primary spectral components of red, green and blue, as shown in Fig. 1.

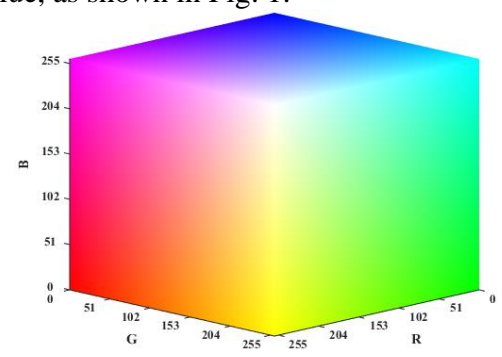

Fig. 1. RGB color cube

\subsection{Basis of image segmentation}

The basic image segmentation is to divide an image into several sub-regions and satisfy the following conditions :

1) ${\underset{i=1}{\mathrm{U}}}_{\mathrm{i}}^{n} R_{i}=R$.

2) $R_{i}$ is a connected set, $i=1,2, \ldots, n$

3) $\forall i, j$, if $i \neq j, R_{i} \cap R_{j}=\varnothing$.

4) $Q\left(R_{i}\right)=$ TRUE , $i=1,2, \ldots, n$, that the pixels in the segment sub-region $R_{i}$ should satisfy the predefined logical attribute $Q$.

* Corresponding author 
5) Any adjacent regions $R_{i}$ and $R_{j}$ satisfy, that is, the $Q\left(R_{i} \mathrm{U} R_{j}\right)=$ FALSE adjacent segment subdomain should not satisfy the same logical attribute $Q$.

Normally, $Q$ is a compound expression. For example, if the average of the pixel gray levels in $R_{i}$ is less than $\mu_{i}$ and the standard deviation is greater than $\sigma_{i}$, then $Q\left(R_{i}\right)=$ TURE

\section{DIMENSIONAL OTSU ALGORITHM}

The segmentation technology for the gray image can be extended to the color image segmentation. We introduce a classic gray image segmentation algorithm based on twodimensional histogram, that is, two-dimensional Otsu algorithm.

For a $M \times N$ image with a gray scale of $L, f(x, y)$ is the intensity of pixel $(x, y), g(x, y)$ is the average intensity of $k \times k$ neighborhood which center is pixel $(x, y)$ :

$$
g(x, y)=\frac{1}{k^{2}} \sum_{m=-k / 2}^{k / 2} \sum_{n=-k / 2}^{k / 2} f(x+m, y+n)
$$

$h_{i j}$ is the number of pixels with $f(x, y)=i$ and $g(x, y)=j$, that is, the frequency of the occurrence of the $(i, j)$, where $i, j=0,1, \ldots, L-1$. Taking a gray image (Fig.2(a)) as an example, the two-dimensional histogram constructed from $i$, $j$ and $h_{i j}$ is shown in Fig. 2 (b). The area corresponding to the two peaks in the figure is the foreground and background in the gray image.

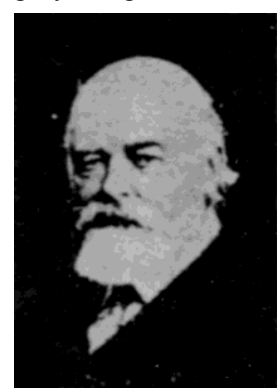

(a) Gray image

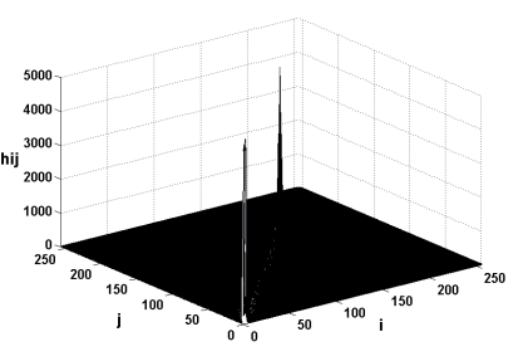

(b) Two-dimensional histogram
Fig. 2. Gray image and its two-dimensional histogram

The combined probability density of $(i, j)$ is:

$$
P_{i j}=h_{i j} /(M \times N)
$$

And $\sum_{i=0}^{L} \sum_{j=0}^{L} P_{i j}=1$. Because of the continuity of image information, the distribution of $p_{i j}$ is mainly concentrated in the range of $(i, j)$ to around the diagonal of $(0,0)$ to $(L-1, L-1)$.

The image is divided into foreground and background ( $C_{0}$ and $C_{1}$ ) by threshold $(i, j)=(s, t)$, the probability of occurrence of the two categories are :

$$
\begin{gathered}
\omega_{0}(s, t)=P_{r}\left(C_{0}\right)=\sum_{i=0}^{s} \sum_{j=0}^{t} p_{i j} \\
\omega_{1}(s, t)=P_{r}\left(C_{1}\right)=\sum_{i=s+1}^{L-1} \sum_{j=t+1}^{L-1} p_{i j}
\end{gathered}
$$

The corresponding mean vectors of the two classes are

$$
\begin{gathered}
\mu_{0}=\left(\mu_{0 i}, \mu_{0 j}\right)^{T}=\left(\sum_{i=0}^{s} \sum_{j=0}^{t} i \frac{p_{i j}}{\omega_{0}}, \sum_{i=0}^{s} \sum_{j=0}^{t} j \frac{p_{i j}}{\omega_{0}}\right)^{T} \\
\mu_{1}=\left(\mu_{1 i}, \mu_{1 j}\right)^{T}=\left(\sum_{i=s+1}^{L-1} \sum_{j=s+1}^{L-1} i \frac{p_{i j}}{\omega_{1}}, \sum_{i=s+1}^{L-1} \sum_{j=s+1}^{L-1} j \frac{p_{i j}}{\omega_{1}}\right)^{T}
\end{gathered}
$$

The total mean vector on the two - dimensional histogram is

$$
\mu_{T}=\left(\mu_{T i}, \mu_{T j}\right)^{T}=\left(\sum_{i=0}^{L-1} \sum_{j=0}^{L-1} i p_{i j}, \sum_{i=0}^{L-1} \sum_{j=0}^{L-1} j p_{i j}\right)^{T}
$$

Since in most cases the distance from the histogram diagonal is negligible, so $\omega_{0}+\omega_{1} \approx 1$ and $\mu_{T} \approx \omega_{0} \mu_{0}+\omega_{1} \mu_{1}$.

Define the dispersion matrix:

$$
S_{b}=\sum_{k=0}^{1} \omega_{k}\left[\left(\mu_{k}-\mu_{T}\right)\left(\mu_{k}-\mu_{T}\right)^{T}\right]
$$

Discrete measure of the target and the background using the trace of the dispersion matrix:

$$
\begin{aligned}
\operatorname{tr} S_{b}(s, t)= & \omega_{0}\left[\left(\mu_{0 i}-\mu_{T i}\right)^{2}+\left(\mu_{0 j}-\mu_{T j}\right)^{2}\right]+ \\
& \omega_{1}\left[\left(\mu_{1 i}-\mu_{T i}\right)^{2}+\left(\mu_{1 j}-\mu_{T j}\right)^{2}\right]
\end{aligned}
$$

When the value of $\operatorname{tr} S_{b}(s, t)$ maximized, $(s, t)$ is the optimum threshold.

Because of the nonuniformity of illumination in the image and the high degree of similarity between the adjacent regions, the hard classification method such as the thresholding method is prone to the wrong segmentation result. Fuzzy set theory is an effective tool for modeling and reasoning on uncertainties. Therefore, this paper uses fuzzy set theory to solve the problem of uncertainty.

\section{BASIS OF FUZZY SETS}

Fuzzy set theory using mathematical methods to describe and deal with the reality of the fuzzy phenomenon. It can make up for the shortcomings of using binary logic alone to describe things. In the fuzzy logic, the membership function is used to reflect the membership degree of the fuzzy set.

Let $A$ be a set on the universe $U$, for any $u \in U$, order

$$
C_{A}(u)=\{1 \text {, 当 } u \in A
$$




\section{0 , 当 $u \notin A$}

Then $C_{A}(u)$ is the membership function of set $A$. The value $C_{A}\left(u_{0}\right)$ of $C_{A}(u)$ at $u=u_{0}$ is called the membership degree of $u_{0}$ to $A$. The closer the value of $C_{A}(u)$ is to 1 , that $u$ belongs to the higher degree of $A$, on the contrary, that $u$ belongs to the lower degree of $A$.

In the application of fuzzy set theory, it is necessary to make a decision by fuzzy comprehensive evaluation of the model. The steps for fuzzy comprehensive evaluation are as follows:

1) Establish the evaluation of the object set $U=\left\{u_{1}, u_{2}, \ldots, u_{n}\right\}$, they used to describe the various attributes of the object.

2) Establish the evaluation set $V=\left\{v_{1}, v_{2}, \ldots, v_{m}\right\}$.

3) Establish a single factor judgment, that is, to establish a modal mapping from $U$ to $F(V)$. The mapping formula is

$$
\begin{gathered}
f: U \rightarrow F(V), \forall u_{i} \in U \\
u_{i} \mid f\left(u_{i}\right)=\frac{r_{i 1}}{v_{1}}+\frac{r_{i 2}}{v_{2}}+\cdots+\frac{r_{i m}}{v_{m}}
\end{gathered}
$$

Where, $0 \leq r_{i j} \leq 1,0 \leq i \leq n, 0 \leq j \leq m$

$$
R=\left(\begin{array}{cccc}
r_{11} & r_{12} & \cdots & r_{1 m} \\
r_{21} & r_{22} & \cdots & r_{2 m} \\
\cdots & \cdots & \cdots & \cdots \\
r_{n 1} & r_{n 2} & \cdots & r_{n m}
\end{array}\right)
$$

$\mathrm{R}$ is a single factor decision matrix, the $(U, V, R)$ constitutes a comprehensive evaluation model.

4) Comprehensive evaluation

Because of the different emphasis on the factors in $U$, we need to assign different weights to each factor, which can be expressed as a fuzzy subset $A=\left(a_{1}, a_{2}, \cdots a_{n}\right)$ on $U$ and $\sum a_{i}=1$.

After $R$ and $A$ are identified, the comprehensive evaluation is:

$$
B=A \circ R
$$

Finally, according to the principle of maximum membership, we make the final decision.

\section{FUSION SEGMENTATION FOR COLOR IMAGE}

In the traditional two-dimensional Otsu segmentation method, the image is divided into two categories $C_{0}(0 \leq i \leq s, 0 \leq j \leq t)$ and $C_{1}(s \leq i \leq L-1, t \leq j \leq L-1)$ by the threshold value of $(s, t)$. Since the adjacent regions have a high degree of similarity at the junction, the pixels near the threshold (Fig. 3 circle area) are of uncertainty, as shown in Fig. 3. At this point the hard decision method is easy to produce the wrong segmentation results. Therefore, this paper uses the membership function to model this uncertainty. Then through the fuzzy comprehensive evaluation method, three color channel information are used for fusion segmentation.

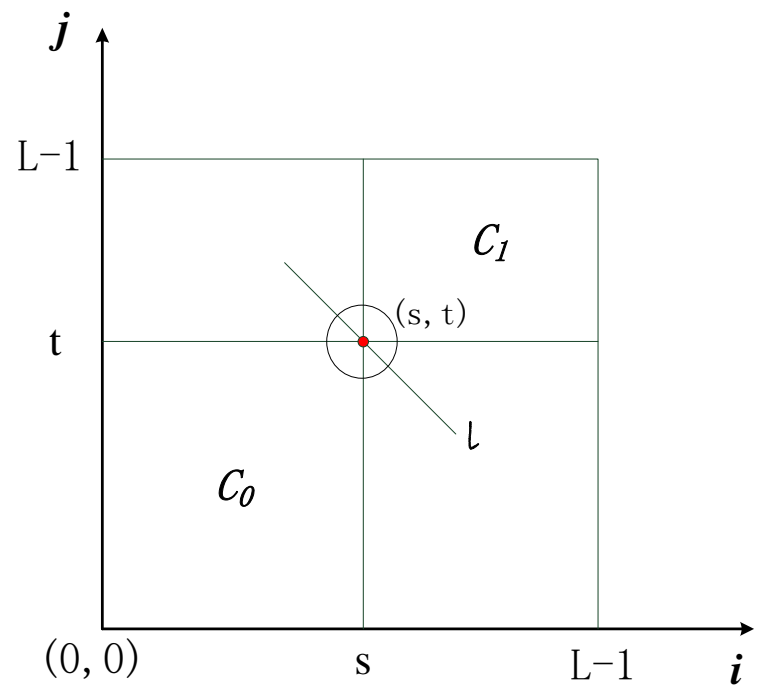

Fig. 3. Segmentation diagram based on two-dimensional histogram

\subsection{Uncertainty modeling}

The color image information from three channels can reflect the pixel category from different angles. Therefore, the problem of color image segmentation can be seen as a multi-attribute decision problem in an uncertain environment.

For pixels located at $(x, y)$, if they are close to threshold $\left(s^{q}, t^{q}\right)$ on color channel $q(q=1,2,3)$, it is difficult to determine whether it belongs to category $C_{0}$ or $C_{1}$. However, if it is in the lower left of the line $l$ on the Fig.3. The pixel $(x, y)$ satisfy formula $\left(f^{q}(x, y)-s\right)+\left(g^{q}(x, y)-t\right)<0$. So the possibility $\left(U_{0}^{q}(x, y)\right)$ of belonging to class $C_{0}$ is large. On the contrary, the possibility $\left(U_{1}^{q}(x, y)\right)$ of belonging to class $C_{1}$ large. We use the following function to express this possibility :

$$
\left\{\begin{array}{l}
U_{0}^{q}(x, y)=\frac{1}{1+e^{-d^{q}(x, y)}} \\
U_{1}^{q}(x, y)=\frac{1}{1+e^{d^{q}}(x, y)}
\end{array}\right.
$$

Which $d^{q}(x, y)=\alpha \times\left[\left(f^{q}(x, y)-s^{q}\right)+\left(g^{q}(x, y)-t^{q}\right)\right]$, $\alpha$ is a constant. The function curve shown in Fig. 4:

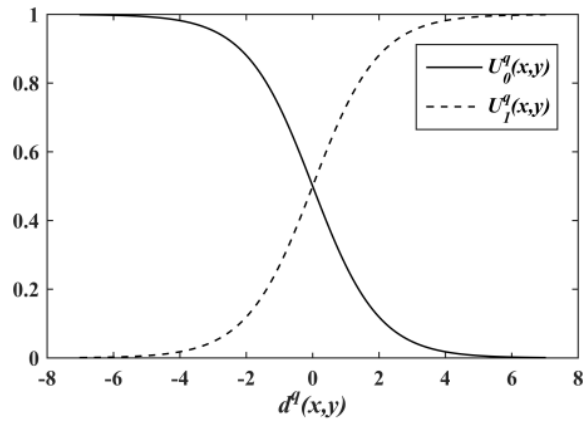

Fig. 4. the function diagram of $U_{0}^{q}(x, y)$ and $U_{1}^{q}(x, y)$ 
According to $U_{0}^{q}(x, y)$ and $U_{1}^{q}(x, y)$, the membership function is established. For the pixels at $(x, y)$, the set of factors for the comprehensive evaluation of the three color channels ( $q=1,2,3)$ information is:

$$
U=\left\{x_{1}, x_{2}, x_{3}\right\}
$$

Where $x_{1}$ is the $\mathrm{R}$ channel pixel class, $x_{2}$ is the $\mathrm{G}$ channel pixel class, $x_{3}$ is the $\mathrm{B}$ channel pixel class.

The comprehensive evaluation of the evaluation set is:

$$
V=\left\{V_{0}, V_{1}\right\}=\left\{(x, y) \in C_{0},(x, y) \in C_{1}\right\}
$$

Where $V_{0}$ and $V_{1}$ respectively indicate that the pixel points are foreground and background.

A single factor judgment matrix is available:

$$
R=\left(\begin{array}{ll}
U_{0}^{1}(x, y) & U_{1}^{1}(x, y) \\
U_{0}^{2}(x, y) & U_{1}^{2}(x, y) \\
U_{0}^{3}(x, y) & U_{1}^{3}(x, y)
\end{array}\right)
$$

\subsection{Fusion segmentation}

Through the uncertainty model, the factor set, the judgment set and the single factor evaluation matrix of the evaluation object are obtained. The weight vector of the comprehensive evaluation is $A=\left(\frac{1}{3}, \frac{1}{3}, \frac{1}{3}\right)$. And the comprehensive evaluation $\mathrm{B}$ is obtained according to the formula (13) to calculate the pixels of each channel The points belong to the membership degree of $C_{0}$ and $C_{l}$.

Finally, according to the principle of maximum membership, the point is classified into a large membership. After each pixel classification is complete, the segmentation ends.

\section{EXPERIMENT}

In order to verify the effectiveness of the proposed method, we selected two color images (Fig. 5(a), (b)) and one optical remote sensing image (Fig. 5(c)) for segmentation experiment. And we compared the results with the traditional twodimensional Otsu thresholding method (The color image is converted into a gray scale image and the threshold segmentation is performed). At the same time, we also compared the use of traditional two-dimensional Otsu thresholding method in the three color channel segmentation results. The experimental results are shown in Fig. 6 to Fig. 8 . In the experiment, when constructing a two-dimensional histogram, the pixel neighborhood average intensity $g(x, y)$ is obtained by $3 \times 3$.

From the results of the experimental, the fusion method in this paper can obtain better segmentation results that compared with the traditional threshold two-dimensional histogram method. And it is better than (or near) the best segmentation result among three channels.

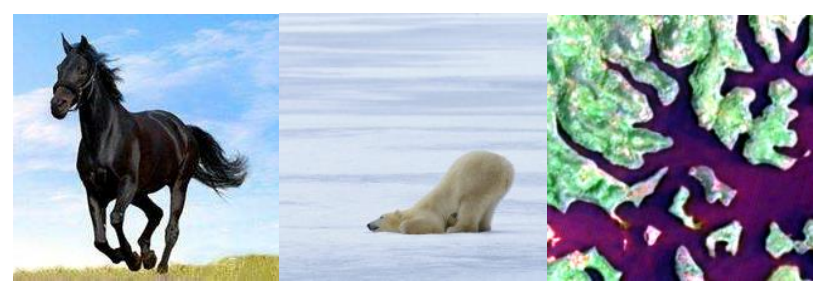
(a) image a
(b) image $b$
(c) image c

Fig. 5. Experimental original image

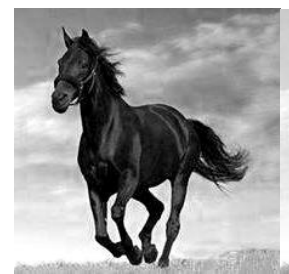

(a)

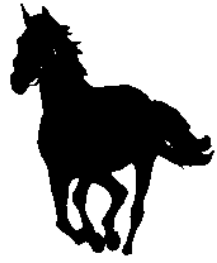

(d)

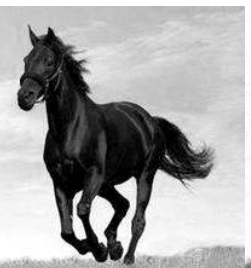

(b)

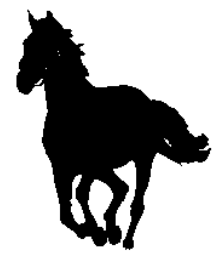

(e)

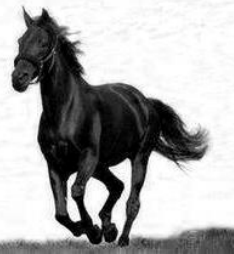

(c)

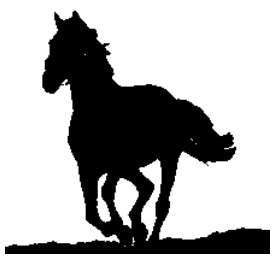

(f)

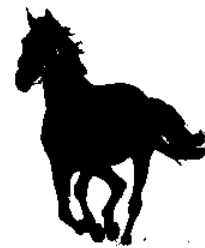

(g)

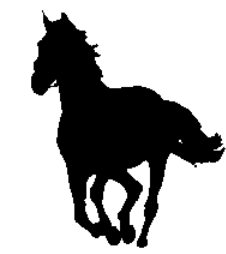

(h)
Fig. 6. Experimental results of image a

(a) R-channel original (b) G-channel original (c) B-channel original (d) R-channel segmentation (e) G-channel segmentation (f) B-channel segmentation (g) Traditional two-dimensional histogram threshold segmentation (h) Segmentation results in this paper

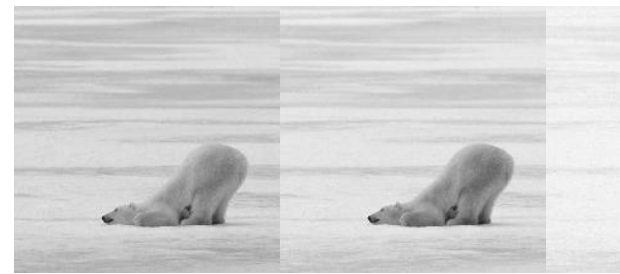

(a) (b)

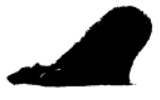

(e)

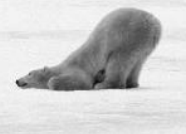

(c)

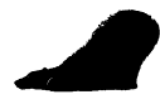

(f)

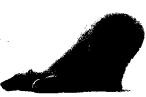

(g)

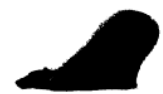

(h)
Fig. 7. Experimental results of image $b$

(a) R-channel original (b) G-channel original (c) B-channel original (d) R-channel segmentation (e) G-channel segmentation (f) B-channel segmentation (g) Traditional two-dimensional histogram threshold segmentation (h) Segmentation results in this paper 


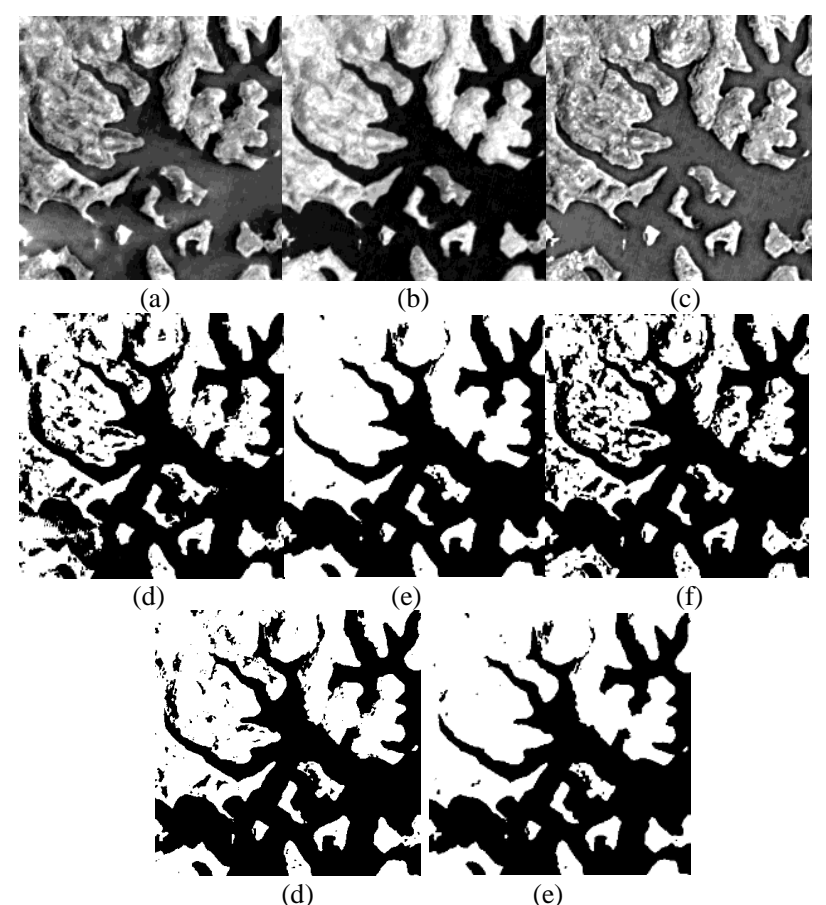

Fig. 8. Experimental results of image $\mathrm{c}$

(a) R-channel original (b) G-channel original (c) B-channel original (d) R-channel segmentation (e) G-channel segmentation (f) B-channel segmentation (g) Traditional two-dimensional histogram threshold segmentation (h) Segmentation results in this paper

\section{CONCLUSION}

In this paper, the fuzzy set theory is used to model the uncertainties in the color image segmentation problem, and the new image fusion segmentation algorithm is designed by using three color channel information. Experiments show that the method proposed in this paper can obtain better segmentation results than the traditional two-dimensional histogram thresholding method. Therefore, this method has important reference value for color image segmentation, and can also provide new ideas for optical remote sensing image and polarization SAR image segmentation.

\section{REFERENCES}

[1] Plataniotis K N, Venetsanopoulos A N. Color image processing and applications[M]. Springer, 2000.

[2] Salem A M. MEDICAL IMAGE SEGMENTATION[J]. International Journal on Computer Science \& Engineering, 2010, 2(4):1209-1218.

[3] Datta R, Joshi D, Li J, et al. Image retrieval[J]. Acm Computing Surveys, 2008, 40(2):1-60.

[4] Alamri S S, Kalyankar N V, Khamitkar S D. Image Segmentation by Using Threshold Techniques[J]. Computer Science, 2010.

[5] Brejl, M, and M. Sonka. "Edge-based image segmentation: machine learning from examples." IEEE International Joint Conference on Neural Networks Proceedings, 1998. IEEE World Congress on Computational Intelligence IEEE, 1998:814-819 vol.2.

[6] Karoui I, Fablet R, Boucher J M, et al. Region-Based Image Segmentation Using Texture Statistics And Level-Set Methods[J]. 2006, 2:II-II.

[7] Bejar H H C, Miranda P A. Oriented relative fuzzy connectedness: theory, algorithms, and its applications in hybrid image segmentation methods[J]. Eurasip Journal on Image \& Video Processing, 2015, 2015(1):21.

[8] Ohtsu N. A Threshold Selection Method from Gray-Level Histograms[J]. Systems Man \& Cybernetics IEEE Transactions on, 1979, 9(1):62-66.

[9] Liu J Z, Li W Q. Automatic thresholding of gray-level pictures via two-dimensional OTSU method[J]. Acta Automatica Sinica, 1993, 19(1).

[10] L.A.Zadeh, Fuzzy sets. Information and Control, 1965, 8: 338 353.

[11] Lehmann I, Weber R, Zimmermann H J. Fuzzy set theory[J]. OR Spectrum, 1992, 14(1):1-9.

[12] Macdonald L W, Mayne C L. Color image processing: US, US5896122[P]. 1999.

[13] Pham D L, Xu C, Prince J L. Current methods in medical image segmentation[J]. Annual Review of Biomedical Engineering, 2000, 2(2):315.

[14] Wang F. Improving remote sensing image analysis through fuzzy information representation.[J]. 1990.

[15] Liu G, Lu X Q. Pixel-Level image Fusion Based on Fuzzy Theory[C]. International Conference on Machine Learning and Cybernetics. IEEE, 2007:1510-1514.

[16] Plataniotis K N, Venetsanopoulos A N. Color image processing and applications[M]. Springer, 2000.

[17] Rajinikanth V, Couceiro M S. RGB Histogram Based Color Image Segmentation Using Firefly Algorithm[J]. Procedia Computer Science, 2015, 46:1449-1457. 